\title{
Level of Detail Continuum for Huge Geometric Data
}

\author{
Florent Duguet* \\ GET/Télécom Paris \\ REVES/INRIA
}
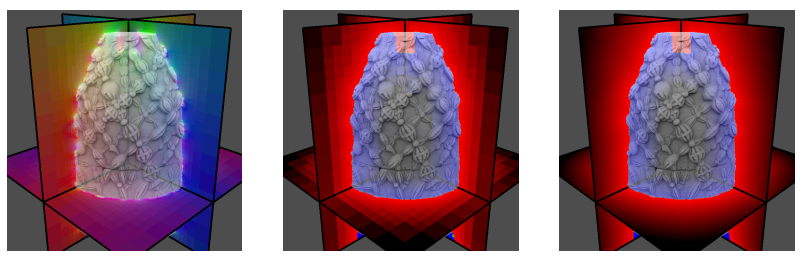

Figure 1: Illustration of our approach. Left, the normals are diffused in the volume defining a guidance vector field. Middle, the distance field. Right, the interpolated distance field.

\section{Introduction}

We propose a unified solution for the creation of levels of detail on very large input data. We build a hierarchical signed distance function in an octree around the data and use this hierarchy to generate a continuum of levels of detail. Our distance function construction, based on the Gradient Vector Flow and the Poisson equation, builds on multigrid resolution algorithms. Using an appropriate interpolation scheme within the octree we obtain a continuous hierarchical distance function, which allows us to define a continuum of levels of detail for huge geometries. During this process, holes and undersampling issues in the input data are automatically corrected. We present three applications of our hierarchy: a novel hierarchical deformable model scheme that can automatically reconstruct closed Eulerian meshes of up to a million faces in a few minutes, an alternate distance-driven contouring approach, and raytracing of huge data models.

\section{Exposition}

To construct levels of detail using previous techniques, it is necessary to combine several different approaches, and to perform three distinct steps. First, an object reconstruction step [Curless and Levoy 1996], followed by a hole filling phase [Davis et al. 2002], and finally a mesh simplification process [Hoppe 1996; Garland and Heckbert 1997]. This three-step approach is cumbersome and costly since the maximal resolution object needs to be built first, and holes at this scale need to be filled.

We propose a unified solution to the creation of a scale space on very large data. We build a signed distance function, which we extend to a large volume around the object, arranged in an octree. The signed nature of this function enables us to easily perform automatic hole-filling and address undersampling issues in the input data. This distance is computed using well known partial differential equations (PDE): the heat and Poisson equations.

Figure 1 illustrates the overview of our approach. First, we build a constrained octree using the input point and normal samples. Second, we diffuse the normals using a Gradient Vector Flow approach such that a smooth vector field is defined in the entire volume around the object. Finally, we extract the potential from this vector field by solving a Poisson equation in the octree. This results in a smooth distance field defined at each cell of the octree. We interpolate node values to provide a smooth distance field in the entire volume around the object. This distance field is made hierarchical using node values. The continuum is defined by interpolating the distance functions of two consecutive clamping levels for the octree. We finally use parametric deformable models (snakes) guided by the hierarchical distance field and

\footnotetext{
*e-mail: Florent.Duguet@sophia.inria.fr

$\dagger$ e-mail: Carlos.Hernandez@enst.fr

‡e-mail: George.Drettakis@sophia.inria.fr

§e-mail: Francis.Schmitt@enst.fr
}
George Drettakis
REVES/INRIA
Francis Schmitt ${ }^{\S}$
GET/Télécom Paris

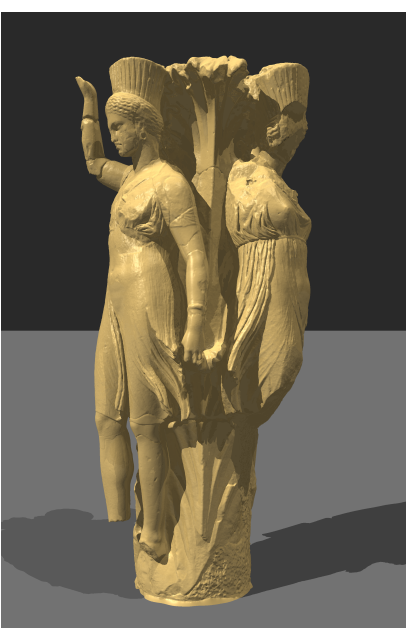

(a)

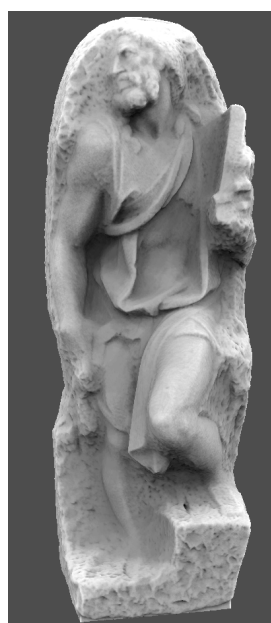

(b)

Figure 2: Left, ray-traced image of the dancers model (47 million input points, image resolution: 1670x3400). Right, the reconstruction of the St.Matthew model using the hierarchical snake algorithm (370 million input points, 1 million triangles).

the guidance vector, improving convergence of previous approaches [Hernandez and Schmitt 2004] by a factor of 100. With this approach, we can build several representations of the same object of increasing complexity very efficiently (about four minutes to build a mesh of 1 million triangles for the St.Matthew model, after distance field construction of 1h40). Results are shown in figure 2 .

\section{Conclusion}

We have presented an algorithm to generate a hierarchical distance function from points and normals. This distance function is continuous, with a continuous gradient, allowing the definition of a continuum of levels of detail. Three applications of our approach are presented: a fast hierarchical surface reconstruction algorithm using deformable models, a distance-driven dual contouring algorithm, and ray-tracing of huge point sets. Since our distance function is signed, any hole or undersampling issue in the input data is automatically corrected. Reconstructed meshes are thus hole-free.

Acknowledgements Data sets thanks to the Digital Michelangelo Project, and Stanford 3D Repository. The dancers column data has been acquired by an Electricité de France R\&D project lead by Guillaume Thibault, involving the Ecole Française d'Athènes, the Ecole du Louvre, and Insight. Thanks also to ACI MD SHOW.

\section{References}

CURLESS, B., AND LEVOY, M. 1996. A volumetric method for building complex models from range images. In Proc. of SIGGRAPH'96, ACM Press / ACM SIGGRAPH, ACM.

DaVis, J., Marshner, S. R., GARR, M., AND LeVoy, M. 2002. Filling holes in complex surfaces using volumetric diffusion. In Symposium on 3D Data Processing, Visualization and Trans.

Garland, M., ANd Heckbert, P. S. 1997. Surface simplification using quadric error metrics. In SIGGRAPH 2000 Proceedings, ACM, 209-216.

Hernandez, C., AND Schmitt, F. 2004. Silhoutte and stereo fusion for 3d object modeling. CVIU 96, 3, 367-392.

Hoppe, H. 1996. Progressive meshes. In SIGGRAPH'96 Proceedings, ACM, 99-108. 